\title{
A HIGH S/N SPECTROSCOPIC SURVEY OF CHEMICAL ABUNDANCES IN DISK POPULATION F0-G2 DWARFS
}

\author{
J. Andersen, Copenhagen University Observatory, Denmark, \\ and Center for Astrophysics, Cambridge, Mass. \\ B. Edvardsson, Uppsala Astronomical Observatory, Sweden \\ B. Gustafsson, Uppsala Astronomical Observatory, \\ and Stockholm Observatory, Sweden
}

P. E. Nissen, Institute of Astronomy, University of Aarhus, DK-8000 Aarhus C, Denmark.

In collaboration with $\mathrm{D}$. Lambert and J. Tomkin high resolution, high $\mathrm{S} / \mathrm{N}$ spectra have been obtained for about 250 disk population F0-G2 dwarfs. The aim of this large programme is to study various abundance ratios as a function of $[\mathrm{Fe} / \mathrm{H}]$ and stellar age. A detailed description of the project and some preliminary results were given by Nissen et al. (1985).

The stars were selected by E. H. Olsen from uvby- $\beta$ photometric catalogues containing all F0-G2 stars brighter than $V=8 \mathrm{~m}$. By means of the $\delta m_{1}$ index these stars were divided into 9 metallicity groups with $[\mathrm{Fe} / \mathrm{H}]$ ranging from -1.0 to 0.3 . Furthermore, the $\delta c_{1}$ index was used to select the somewhat evolved stars, such that ages could be estimated. Of these stars the 30 brightest in each metallicity group were put on the observing programme.

The northern programme stars have been observed with the McDonald Observatory $2.7 \mathrm{~m}$ telescope, Coudé spectrograph and Reticon detector, and the southern stars with the ESO $1.4 \mathrm{~m}$ CAT telescope and Coude Echelle Spectrometer. For 10 stars that have been observed from both observatories the corresponding two sets of equivalent widths agree within $\pm 3 \mathrm{~mA}$.

Until now equivalent widths have been measured for 57 southern stars, and a preliminary LTE model-atmosphere analysis of the data has been carried out as described by Nissen et al. (1985). The parameters, $T_{\text {eff }}, g$ and overall metal abundance of the model atmosphere corresponding to a given star, are estimated from the uvby- $\beta$ photometry. The analysis is differential with respect to the Sun in the sense that gf-values of the lines are derived from solar equivalent widths as measured in the spectrum of reflected sunlight.

In the figures some results of the abundance survey are shown. The following trends are seen: 
i) The $\mathrm{O} / \mathrm{Fe}$ ratio increases with decreasing iron abundance according to the formula $[\mathrm{O} / \mathrm{Fe}]=-0.5[\mathrm{Fe} / \mathrm{H}]$.

ii) The elements $\mathrm{Mg}$ and $\mathrm{Si}$ are overabundant with respect to iron for $[\mathrm{Fe} / \mathrm{H}]<-0.6$. A similar effect is present for $\mathrm{Ca}$ and $\mathrm{Ti}$. [Si/Fe] shows a significant offset from zero at $[\mathrm{Fe} / \mathrm{H}]=0.0$.

iii) Aluminium shows an enhanced odd-even effect with respect to magnesium for $[\mathrm{Fe} / \mathrm{H}]<-0.3$. A similar well-defined effect is not found for sodium, but the scatter in $[\mathrm{Na} / \mathrm{Fe}]$ tends to increase for $[\mathrm{Fe} / \mathrm{H}]<-0.3$.
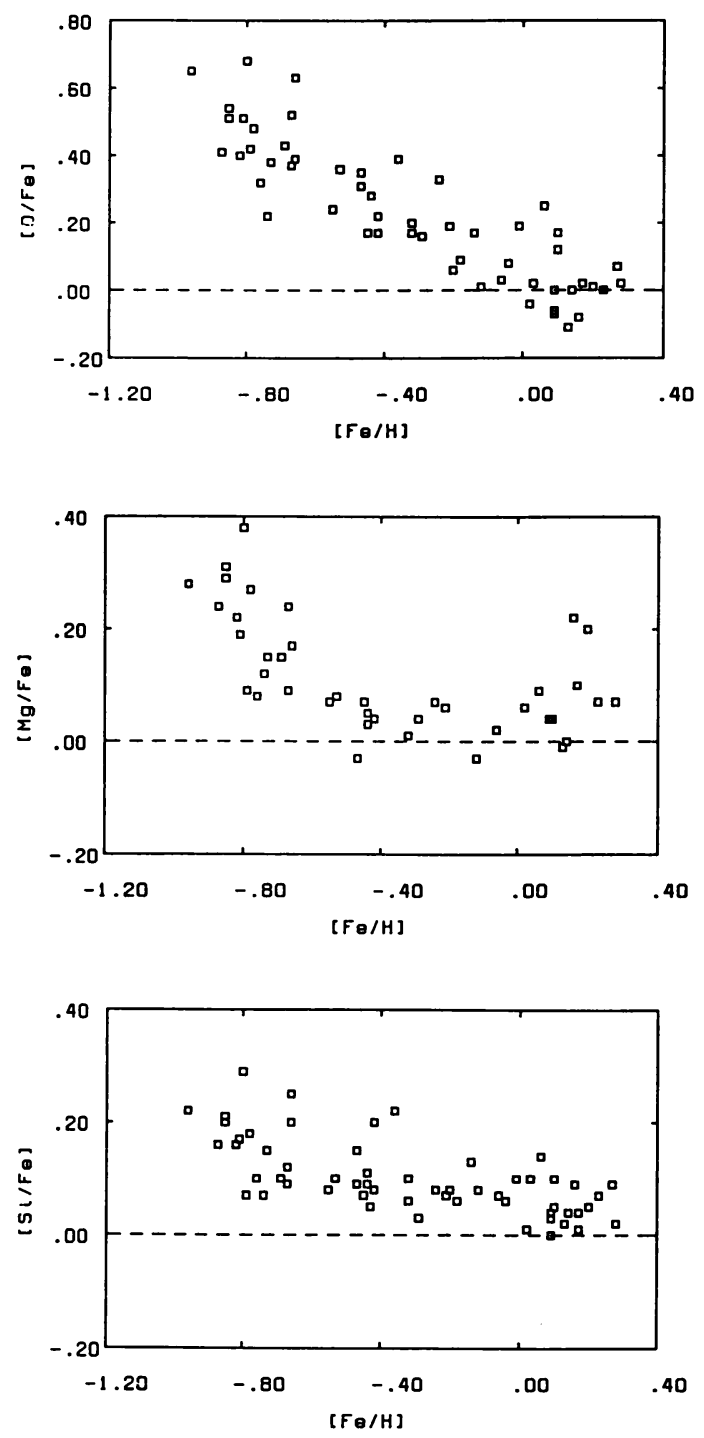

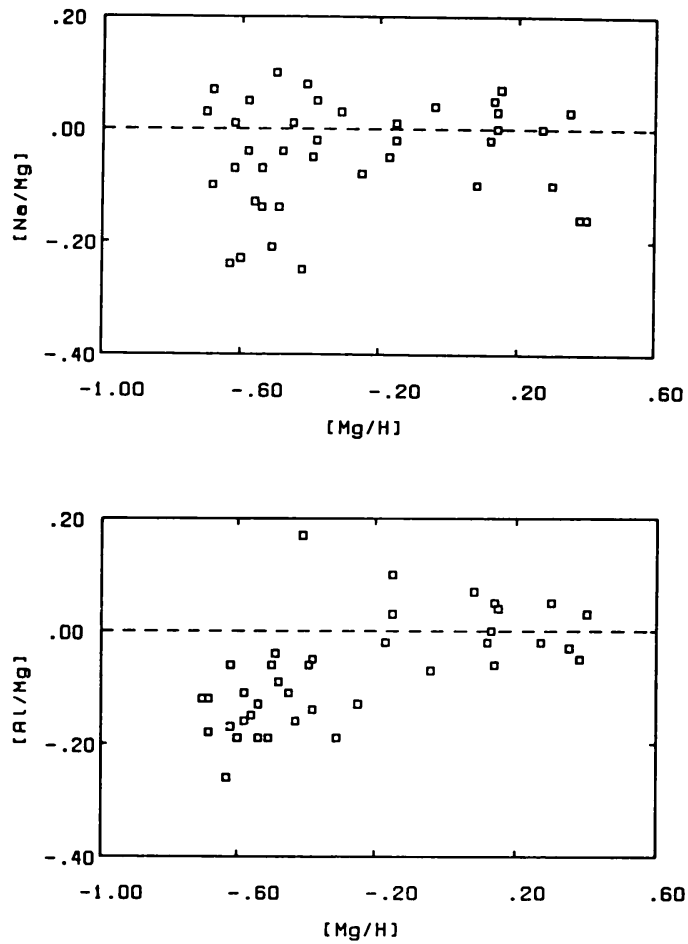

These trends should not be taken too seriously until the LTE analysis has been replaced by non-LTE computations, which are presently being carried out, and the effects of convection and inhomogeneities have been scrutinized.

The most important conclusion at present is:

All abundance ratios with respect to iron have a surprisingly small scatter at a given $[\mathrm{Fe} / \mathrm{H}]$. The $\mathrm{rms}$ dispersion is \pm 0.10 for $[\mathrm{O} / \mathrm{Fe}], \pm 0.04$ to \pm 0.06 for the $\alpha$-elements, and \pm 0.05 to \pm 0.10 for $[\mathrm{Al} / \mathrm{Fe}]$ and $[\mathrm{Na} / \mathrm{Fe}]$. This is the kind of scatter one would expect from the errors in the analysis and in the equivalent widths. Thus, there is no evidence for any cosmic scatter (except maybe in the case of $\mathrm{Na} / \mathrm{Fe}$ for the metal-poor stars). In particular the small scatter in the $\mathrm{N}_{\alpha} / \mathrm{N}_{\mathrm{Fe}}$ ratio (less than $10 \%$ ) at a given $[\mathrm{Fe} / \mathrm{H}]$ is remarkable. Either the $\alpha$-elements and iron are produced in constant proportions at a given time by the different supernovae contributing to the nucleosynthesis or the ejecta of supernovae are very well mixed with the interstellar gas before star formation takes place.

\section{REFERENCE}

Nissen P.E., Edvardsson B., Gustafsson B., Proc. ESO workshop on "Production and Distribution of $C, N, O$ Elements", Eds. E.J. Danziger, F. Matteucci and K. Kjär, p.131. 